\title{
Propuesta del Nuevo Modelo Didáctico Aplicado en Construcción
}

\author{
BEGoña BLANdón GONZÁLEZ \\ Departamento Construcción Arquitectónica 1 \\ Escuela Técnica Superior de Arquitectura. \\ Universidad de Sevilla \\ bblandon@us.es \\ ORCID: https://orcid.org/0000-0003-1025-5675 \\ D.O.I.: http://dx.doi.org/10.12795/JDU.2018.i01.03 \\ Pp.: $58-79$
}

\section{Resumen:}

La presente comunicación se desarrolla como parte del Programa de Formación e Innovación Docente del Profesorado 2018. Se propone el Ciclo de Mejora Docente (CMD) en la asignatura de Construcción 2 perteneciente al Grado en Fundamentos de la Arquitectura de la Universidad de Sevilla.

En esta asignatura, el carácter teórico-práctico de los objetivos docentes, presentan la oportunidad de aplicar esta experiencia, introduciendo un formato de clases más dinámico, en el que docente y estudiantes interactúen compartiendo la responsabilidad del aprendizaje. Durante el diseño del CMD propuesto, se profundiza en los contenidos esenciales y el desarrollo de las sesiones en el aula.

Los resultados obtenidos muestran importantes mejoras en las calificaciones del estudiante. El diseño propuesto ha supuesto un importante avance no solo en la progresión del aprendizaje sino en la seguridad 
con que el estudiante se enfrenta a una prueba de evaluación, lo que ha llevado a considerar el éxito en esta primera convocatoria.

Palabras clave: Construcción, Arquitectura, Ciclo de Mejora, Innovación Docente, Docencia Universitaria

\section{Interés y Objetivos}

\section{Contexto de la intervención}

Con la llegada del nuevo marco de referencia europeo y los cambios sustanciales experimentados en los nuevos planes de estudio, surge la oportunidad de revisar el sistema de docencia impartido hasta hoy siendo necesario, por parte del docente, la búsqueda de nuevas herramientas que agilicen su actividad acercándose a los importantes cambios sociales, intereses y capacidades que presenta el auditorio que las recibe. Es el momento, por tanto, de introducir un nuevo formato de clase más dinámico, en el que docente y estudiantes interactúen compartiendo la responsabilidad del aprendizaje, lo que hace inevitable la correcta gestión del tiempo dentro y fuera del aula.

En esta titulación, las distintas asignaturas de construcción se imparten en cada curso académico del Grado. Así, la formación obligatoria del área de Construcción Arquitectónica se reparte en 6 asignaturas de 6 créditos ects de formación obligatoria (60 horas docentes + 90 horas no presenciales) cada una y, en los Talleres de Arquitectura, compartiendo docencia con otras asignaturas.

En los primeros cursos del Grado, las asignaturas de construcción se centran en la adquisición de competencias, entre las que se pueden destacar la capacidad para aplicar los conocimientos al trabajo de una forma profesional, la resolución de problemas, capacidad para interpretar datos y el razonamiento crítico, la habilidad gráfica, visión espacial o intuición mecánica. 
La asignatura de Construcción 2, de segundo curso del Grado, desarrolla su línea de contenidos centrada en el diseño de los sistemas constructivos: estructuras, cimientos y envolventes del edificio (cerramientos y cubiertas). En cada uno de ellos, se incluye la definición de materiales y productos así como un primer acercamiento al cálculo y pre-dimensionado de elementos que permitan el diseño correcto de las soluciones adoptadas para el edificio. En general, las líneas de contenido se desarrollan en ámbitos muy relacionados pudiendo considerarse como complementarios y, por tanto, debiendo mantener en todo momento un aprendizaje paralelo entre temas.

Durante el curso 2017, como parte del Curso de Iniciación del Programa de Formación e Innovación Docente del Profesorado, se inició una parte del diseño de la docencia en la asignatura de Construcción 2. Durante ese periodo, se trabajaron los contenidos impartidos correspondientes al tema de cimientos y la metodología docente en el aula (hasta la fecha realizada de forma tradicional).

En el nuevo curso 2018, se toma como punto de partida ese modelo inicial revisándolo y haciéndolo extensible a todo el temario de la asignatura de Construcción 2. Así, se presenta un CMD más amplio que profundiza en el diseño de las sesiones en base a las necesidades detectadas en el aula y los contenidos esenciales de la materia. Este diseño experimental se realiza durante 32 horas, repartidas en 8 sesiones de 4 horas cada una y centradas en los temas que forman parte de la asignatura.

\section{Dificultades encontradas en el aprendizaje de la asignatura}

En las disciplinas técnicas, los estudiantes deben asimilar y aplicar conceptos clave de gran complejidad antes de poder aplicarlos de forma más o menos gráfica. A menudo, incluso los conceptos más básicos presentan dificultades de aprendizaje, lo cual puede traer como 
consecuencia el fracaso en la comprensión de conceptos más avanzados y, lógicamente, la imposibilidad de aplicar en la práctica lo aprendido.

El estudio de las dificultades en el aprendizaje que estudiantes y profesores encuentran en la asignatura de Construcción 2 y las causas que pudieran provocarlas, contribuyen en el diseño de estrategias que facilitan el aprendizaje. A este respecto, las trabas como el nivel de acceso en la formación gráfica y tecnológica que presentan los estudiantes en los primeros cursos del Grado, muestran la realidad existente y dificultan el desarrollo que cada asignatura requiere para alcanzar los objetivos docentes definidos en el correspondiente Programa Docente. Por otra parte, la dificultad de los contenidos, el ritmo acelerado en las explicaciones del profesor, la falta de perspectiva profesional y entorno compartido con otras disciplinas de igual relevancia, se destacan como enemigos del aprendizaje y hace necesario la búsqueda de recursos que favorezca el seguimiento de la asignatura impidiendo que los estudiantes dejen de asistir a clase después de las primeras semanas de curso. Entre las características que se presentan en el auditorio de estudiantes matriculados en la asignatura de Construcción 2 (en base al estudio realizado en las asignaturas de Construcción 1 y Construcción 2, en los grupos 1.01, 1.05 y 2.04 durante los años 2012 a 2017), se destacan algunas de las carencias que impiden el máximo rendimiento del aprendizaje:

1. El primer contacto en materia de construcción se realiza en primer curso, con un programa de la asignatura de Construcción 1 que se organiza en excesivos temas, de extenso contenido que se imparten con un ritmo acelerado lo que imposibilita, al estudiante, la posibilidad de asimilar adecuadamente la materia impartida.

2. La asignatura de Construcción 2 se desarrolla en el segundo cuatrimestre de segundo curso y la asignatura de Construcción 1 se imparte en el primer cuatrimestre de 10 curso, lo que supone un espacio de tiempo entre ambas de 15-16 meses con unas vacaciones de verano entre ellas. Este 
periodo entre ambas asignaturas deriva en el olvido de todo lo que pudieron memorizar en su momento lo que obliga a dedicar un tiempo en el aula a recuperar estos conocimientos. Es destacado observar cómo, los repetidores de Construcción 1, examinados en la convocatoria de diciembre o febrero de ese mismo curso, son los que se incorporan a Construcción 2 con un nivel de conocimientos que les permite avanzar en la asignatura de la forma prevista.

3. La estructura del conocimiento en construcción, contiene una base importante de otras áreas, Acondicionamiento, Instalaciones, Estructuras y Expresión Gráfica, disciplinas que se hacen imprescindibles para el aprendizaje completo y como parte del diseño final del edificio, la falta de referencias al respecto, lleva a un aprendizaje por bloques independientes, muy alejado de la práctica profesional.

4. Asimismo, la coincidencia de la entrega final de Construcción 2 con las entregas correspondientes a otras asignaturas (para aspirantes a la evaluación continua, la asignatura establece una entrega oficial de la Práctica de Curso y supone un $40 \%$ de la nota del estudiante en la primera convocatoria), frena el desenlace del curso mostrando en su tramo final un alto porcentaje de abandono.

5. La organización de horarios con sesiones de 4 horas de duración supone un importante descenso en el nivel de atención que presta el estudiante cuando desconecta en la "lección magistral" tradicional. Se hace necesario buscar actividades que permitan agilizar las horas de clase, evitar la monotonía e impulsar el aprendizaje.

6. Finalmente, la necesidad de cumplir con la planificación prevista en el cuatrimestre, lleva a forzar las expectativas sobre los resultados del estudiante confiando en su aprendizaje no 
presencial y su mayor o menor habilidad en la gestión del tiempo y actividades.

Frente a esos problemas o irregularidades detectadas, se han planteado los principios didácticos sobre los que se basa el diseño experimental propuesto en la asignatura.

\section{Diseño del CMD}

\section{Principios Didácticos}

Los principios didácticos en los que se basa el diseño experimental de la asignatura consideran para la mejora en el aprendizaje del estudiante, el contenido de la enseñanza, el modelo metodológico utilizado y la evaluación final como objetivos de la intervención. Con ello, se persigue alcanzar:

- Mayor participación del estudiante en su propia formación: Para ello, la docencia se presenta como respuesta a cuestiones de interés para su práctica profesional que el estudiante debe resolver dentro y/o fuera del aula (asimismo deberá guiarse el trabajo no presencial desde el aula).

- Generar y mantener la curiosidad del estudiante: El aprendizaje será continuo durante las sesiones, conceptos más teóricos dosificados, sin distinguirlos de la práctica, mediante la secuencia de preguntas y su respuesta posterior.

- Clases que deben ser más interactivas: Se buscará en todo momento la participación del estudiante en el aula con la búsqueda de información y resolución de problemas que se gestiona con actividades prácticas.

- Reducir la línea individualista del aprendizaje: Las actividades se desarrollarán en grupos de trabajo (2-3 estudiantes) con datos de interés para cada grupo. Los distintos resultados llevarán a incluir puestas en común y reflexión final. 
- Incluir Mapas de Contenido en las explicaciones: Se desarrollarán en la pizarra durante la secuencia de preguntas, posibles aclaraciones y resultados de las actividades. Estos esquemas gráficos, se mantendrán durante toda la sesión a fin de ir completándose durante la jornada.

- Relacionar contenidos con otras áreas de interés: Se facilitará el recuerdo de lo ya aprendido en cursos y temas anteriores con enlaces, referencias, apuntes y resúmenes como complemento a las clases.

- Valorar la progresión en el aprendizaje distinguiendo la evaluación del progreso, las metas alcanzadas, y la calificación final.

\section{Nuevo Modelo Metodológico propuesto}

Se propone un modelo metodológico en la Figura 1 en el que se diferencian tres fases de trabajo paralelas al proceso de aprendizaje que se espera. El desarrollo docente se apoya en la futura práctica profesional del estudiante extrayendo de ésta un caso práctico con el que se inicia el modelo. Durante la revisión de los resultados surgirá una secuencia de preguntas inmediatas que permiten el desarrollo ordenado de la docencia y a partir de las cuales se extraen los contenidos esenciales de cada tema. De forma cíclica, la resolución de nuevas incógnitas supondrá el desarrollo de nuevas actividades y la extracción de los nuevos contenidos de interés. 


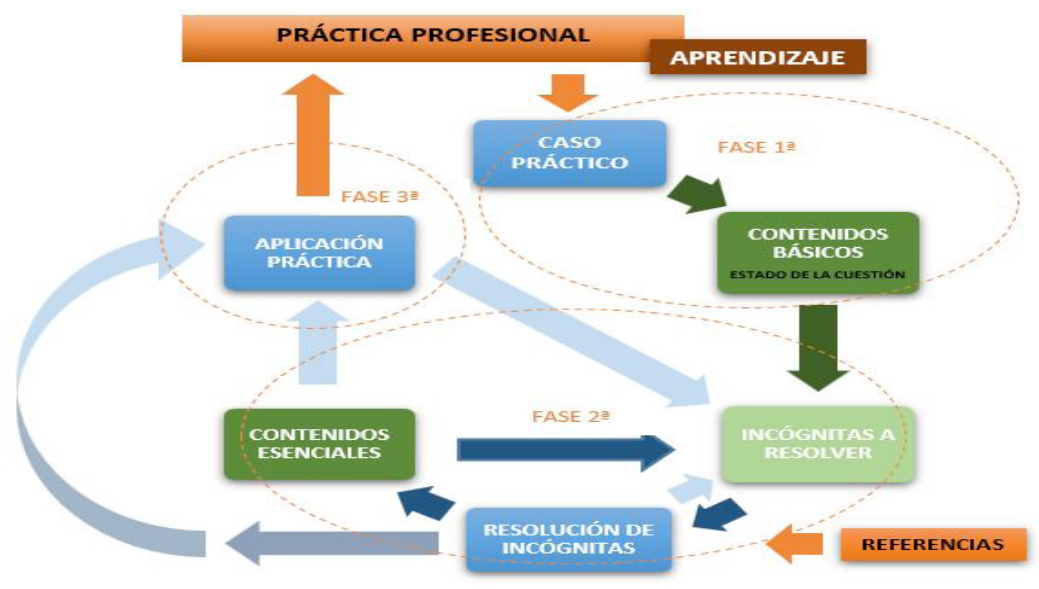

Figura 1. Nuevo Modelo Metodológico propuesto.

En este modelo, se incorpora una mayor dedicación en el aula a las actividades prácticas manteniendo la curiosidad del estudiante y su implicación en la resolución de cuestiones sobre un caso práctico que lo acerca a su futura práctica profesional. Las explicaciones teóricas son las solicitadas y el contenido de los apuntes publicados por la asignatura serán solo datos y complemento conceptual. Respecto a la aplicación práctica de lo aprendido, se desarrollará de forma individual y tomando como objeto la Práctica de Curso. Este modelo presentado guiará el desarrollo de las sesiones propuesto en el siguiente apartado.

\section{Contenido de las sesiones}

¿Qué contenidos quiero enseñar?: A partir de los Principios Didácticos mostrados, se han jerarquizado y priorizado los contenidos de cada tema destacando los esenciales y distinguiendo conceptos, procedimientos y actitudes. Para ello, se plantean cuatro preguntas principales, extensibles a todos los sistemas constructivos, que permiten diseñar las sesiones:

Jornadas de Formación e Innovación Docente del Profesorado I № 1 (2018) Esta obra se distribuye con la licencia Creative Commons 
1. ¿Para qué sirve?: Finalidad y uso del sistema o elemento constructivo objeto del estudio, necesidad en el edificio, contexto.

2. ¿Qué elementos lo integran?: Requisitos según su objetivo en el edificio, partes que lo componen, exigencias a los materiales/productos utilizados: mortero, hormigón, ladrillo, etc.

3. ¿Cómo los diseño?: Objetivos, exigencias normativas, tipos/clasificaciones derivadas, transmisión de cargas/acciones, predimensionado y armado, proceso de ejecución en obra, etc.

4. ¿Me interesa en mi práctica profesional?: Utilidad del tema, contenido de un Proyecto de Ejecución, memoria y planos, puesta en obra y control de calidad.

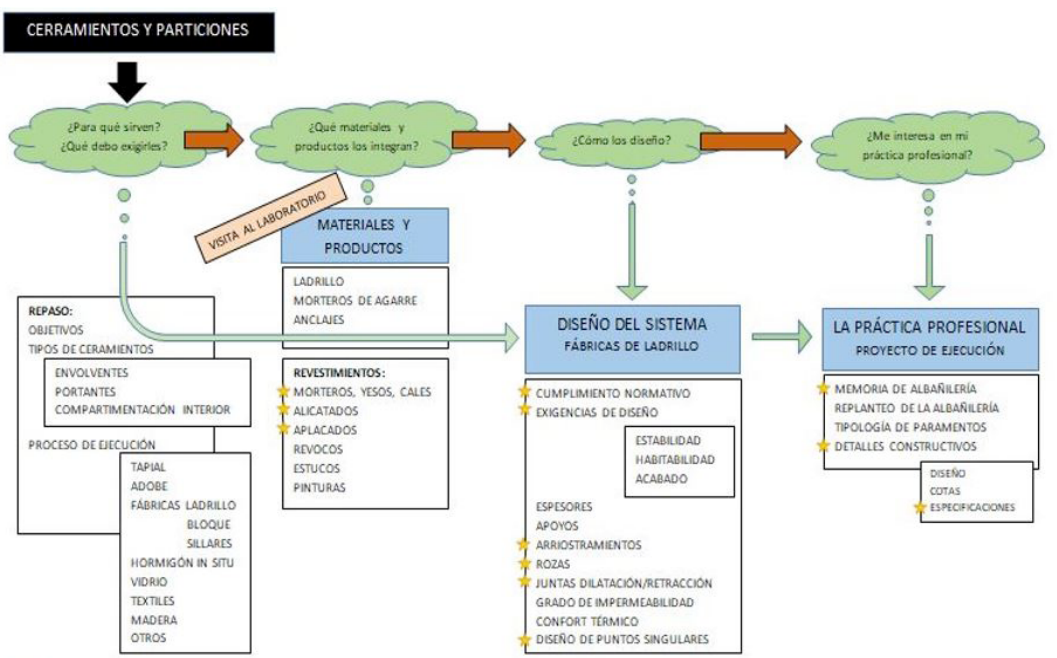

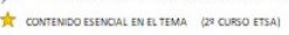

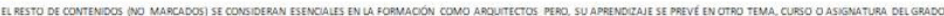

Figura 2. Mapa de Contenidos del tema Cerramientos y Particiones Interiores.

Estas cuatro cuestiones derivan en otras secundarias que generan actividades concretas cuya resolución permite profundizar en el aprendizaje de los conocimientos

Jornadas de Formación e Innovación Docente del Profesorado I № 1 (2018)
Esta obra se distribuye con la licencia Creative Commons

Reconocimiento-NoComercial-SinObraDerivada

Internacional (CC BY-NC-ND 4.0.) 
esenciales. Las actividades previstas tratan de impulsar las habilidades existentes en el estudiante reforzando su posibilidad o capacidad creativa, por ello, tanto en el desarrollo como en la exposición y puesta en común posterior, se opta por actividades que contengan un alto lenguaje y contenido didáctico en estas vertientes.

\section{Desarrollo de las sesiones}

La estructura seguida para el desarrollo de las clases ha supuesto una adecuada combinación entre actividades prácticas y contenidos más teóricos. El contenido de cada tema se desarrolla a lo largo de $2-3$ sesiones de 4 horas de duración (2+descanso+2). Como puede apreciarse en la Figura 3, el nuevo modelo metodológico propuesto guiará el desarrollo de cada sesión diferenciando las tres fases de trabajo consideradas para este aprendizaje:

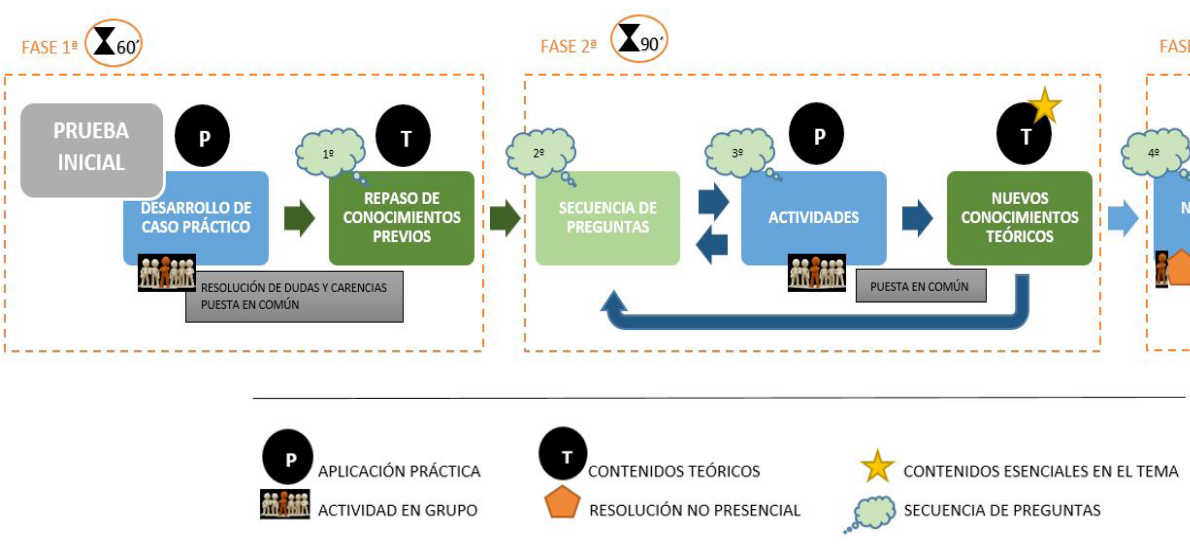

Figura 3. Esquema general del desarrollo de las sesiones.

Fase 10- Espacio y tiempo destinado al REPASO DE CONOCIMIENTOS:

En la primera sesión de un tema, se reparte la PRUEBA DE INICIO a fin de conocer los conocimientos con los que el estudiante se incorpora. Esta prueba puede incluir CASOS A ESTUDIO sobre uno o varios edificios existentes tratando 
de reconocer aquellas soluciones constructivas correspondientes al tema y cuya revisión de errores o consulta de dudas durante el desarrollo de la actividad facilita el repaso de los conocimientos básicos adquiridos en primer curso.

En el resto de sesiones del tema, esta primera parte se destina la revisión y supervisión de los trabajos encargados en la sesión anterior (llamamos PRÁCTICAS 1,2,3). En la pizarra se comentan los errores más repetidos, se repasan criterios de corrección y resuelven dudas generales surgidas durante el aprendizaje no presencial. El estudiante corrige su práctica (detectando sus propios errores), posteriormente y, de forma individualizada, se resuelven las dudas o preguntas particulares que hayan quedado y se califica el trabajo.

En esta etapa se inicia el desarrollo del MAPA DE CONTENIDOS del tema en la pizarra. A lo largo de la jornada y el resto de sesiones se irá completando de forma paralela al desarrollo del temario.

Fase 2-- SECUENCIA DE PREGUNTAS y resolución:

A partir del repaso previo inicial surgen incógnitas que se resuelven en el aula con actividades grupales en las que, durante su desarrollo y/o conclusión (15-30 minutos), se permite la reflexión y extracción de los contenidos esenciales de la asignatura en este punto (llamamos ACTIVIDADES 1,2,3). La puesta en común de los resultados y la exposición de nuevos casos por parte del estudiante, deriva en nuevas cuestiones de interés y permite disponer de una casuística variada sobre distintas soluciones que acercan su formación a la futura práctica profesional. En esta etapa se profundiza en los contenidos teóricos esenciales de la asignatura (aspectos de diseño, predimensionado y armado de las soluciones previstas).

Jornadas de Formación e Innovación Docente del Profesorado I № 1 (2018) Esta obra se distribuye con la licencia Creative Commons 
¿Qué sistemas estructurales me rodean?

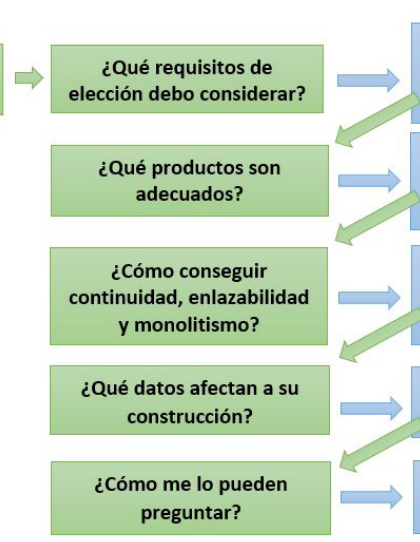

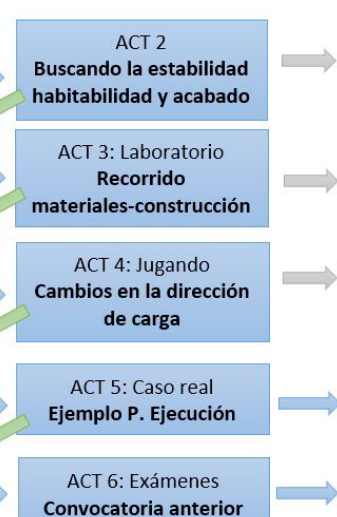

Convocatoria anterior

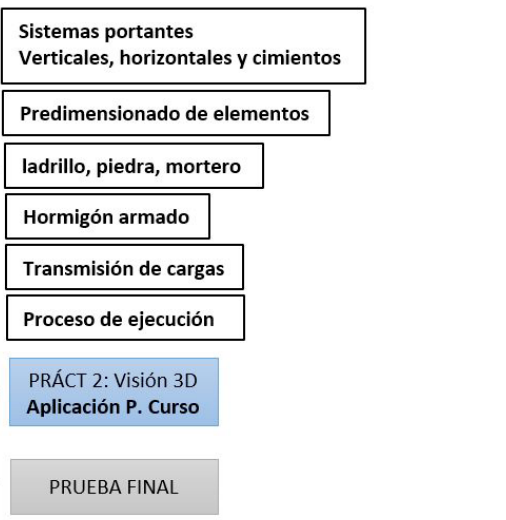

Sistemas portantes

Figura 4. Relación de cuestiones y actividades en el tema Estructuras.

Fase 3-- APLICACIÓN PRÁCTICAy PRÁCTICA PROFESIONAL:

Extraídos y expuestos los contenidos esenciales del tema de forma grupal, el estudiante debe enfrentarse a ellos de forma individual con el desarrollo de la Práctica de Curso (PRÁCTICAS 1,2,3). Esta actividad se inicia en el aula ( \pm 30 minutos) y puede finalizarse en casa (a fin de evitar el abandono de la asignatura por la coincidencia de la entrega final con las entregas de otras asignaturas del curso, se organizan las revisiones semanales, lo que supone entregas más reducidas y un proyecto de ejecución final más controlado).

El interés por el desarrollo de este tipo de prácticas, se anima destinando tiempo a la muestra de un PROYECTO DE EJECUCIÓN ya visado. Como objetivo, relacionar la actividad profesional con el aprendizaje (20 minutos).

Al final de cada tema el estudiante resuelve, de forma individual, una PRUEBA FINAL que incluye las preguntas de la prueba Inicial e incorpora otras nuevas de contenido teórico-práctico sobre las actividades desarrolladas en el aula en el transcurso de las últimas sesiones. Esta prueba se entrega de forma anónima y permite valorar el aprendizaje del estudiante y su progreso, así como los contenidos no asimilados o que presentan dificultades ( \pm 20 minutos). 
Como conclusión al tema, se destinan 20 minutos a la muestra de EXÁMENES de cursos anteriores a fin de comprobar cómo se les puede evaluar/preguntar en una convocatoria, lo que han hecho en modo práctico.

\section{Aplicación del CMD}

\section{Valoración sobre el desarrollo de las sesiones}

Respecto al CMD desarrollado en 2017 sobre el tema de cimientos, se ha superado la dificultad encontrada sobre la necesidad de generar el interés en el estudiante por trabajar en el aula y no solo escuchar (algunos asistentes abandonaron el aula al iniciar el ciclo y comprobar que la clase se desarrollaría de este modo). Durante el curso 2018, la dinámica de las sesiones presentada desde el inicio del cuatrimestre no ha dado otra opción de trabajo al estudiante y, su participación e implicación en el aprendizaje ha formado parte de la asistencia. Por otra parte, las actividades en grupo y la continua relación con "su propio edificio" (objeto de la Práctica de Curso), han favorecido la puesta en común negando la posibilidad de que alguno se mantuviese al margen.

Durante la aplicación del CMD propuesto se han podido extraer algunas reflexiones sobre el desarrollo de las clases. Entre los aciertos:

- El orden establecido en el desarrollo de las sesiones las ha uniformado y plantea una secuencia de trabajo diaria que, aunque repetitiva, ha generado la confianza en el estudiante.

- La resolución de dudas de nivel básico a principio de cada tema, aunque inicialmente puede ralentizar el avance en la materia, supone una aceleración posterior al no tener que volver atrás para aclarar ciertos vacíos de base. Asimismo, dedicar un tiempo para el 
repaso del día anterior (aunque a modo de lección magistral, revisando los errores más comunes y corrigiendo las prácticas entregadas) ha permitido repasar, asimilar lo aprendido e introducir un avance de la nueva materia.

- Se ha conseguido una jornada más interactiva con la participación de todos los asistentes y los estudiantes han podido compartir y enriquecerse con la experiencia de los demás.

- En general no ha hecho falta forzar el ritmo para ajustarse al tiempo de clase en las actividades porque no se han proyectado gran parte de los P. Point del tema.

- La incorporación de defectos y lesiones en edificios como parte del contenido visual proyectado en las sesiones ha generado el interés por la prevención y el diseño correcto de los sistemas constructivos aplicando la normativa.

- Las entregas semanales sobre la Práctica de Curso han reducido el volumen de trabajo gestionado por el estudiante en las horas no presenciales y permite al docente conocer la progresión en el aprendizaje facilitando el seguimiento individual y su evaluación continua.

- La muestra de exámenes correspondientes a convocatorias y cursos anteriores al finalizar el tema, ha reforzado en el estudiante la confianza de haber recibido la materia y la relevancia de los conocimientos esenciales sobre los que se ha trabajado.

- Respecto a las referencias, tan necesarias en ámbitos paralelos como instalaciones, estructuras, etc, este curso se ha puesto en marcha, como resultado del III Plan Propio de Docencia en la Convocatoria de Apoyo a la Coordinación e Innovación Docente, un sitio web diseñado para la tutorización de las horas de aprendizaje no presencial que facilita al estudiante la búsqueda de referencias y permite complementar 
el desarrollo de las actividades con los enlaces relacionados.

- Respecto a las dificultades encontradas, la mayor parte se localizan en las propias actividades desarrolladas en el aula:

- No se han entregado por escrito los enunciados y objetivos de las actividades, se han dictado datos previos e información adicional. Como consecuencia, ha resultado excesiva la implicación del profesor durante el desarrollo del trabajo, interrumpiendo para aclaraciones o repeticiones y los estudiantes no han trabajado su autonomía.

- Las actividades de cálculo requieren el uso de la normativa. Para evitar retrasos en la planificación de las sesiones se ha dedicado tiempo a la búsqueda de tablas y directrices. la proyección de pantallazos y de los P. Point de la asignatura, ha acelerado el ritmo pero entorpecido la concentración y la dinámica ha resultado muy desordenada en el aula.

- El uso de los ordenadores como instrumento de trabajo para presentar las practicas ha llevado a excesivas imitaciones y coincidencia en los resultados entre los estudiantes.

Como conclusión a estas incidencias detectadas se proponen en el punto 4 nuevas actuaciones para un nuevo diseño experimental en la docencia que complete el actual.

\section{Evaluación del progreso de aprendizaje}

Al inicio y final de las sesiones, correspondientes a cada tema, se han realizado las pruebas de aprendizaje que hacen posible concretar el nivel de conocimientos adquirido por el estudiante tras la aplicación del CMD propuesto. Para ello, a partir de la jerarquización de los contenidos de la asignatura, ha sido posible plantear un diagrama en escalera que muestra los distintos niveles en la progresión del aprendizaje en los que puede situarse al 
estudiante. Los porcentajes indicados en la Figura 5 reflejan el nivel en el que se encuentran los estudiantes de la asignatura antes y después de la aplicación del CMD y su calificación correspondiente.

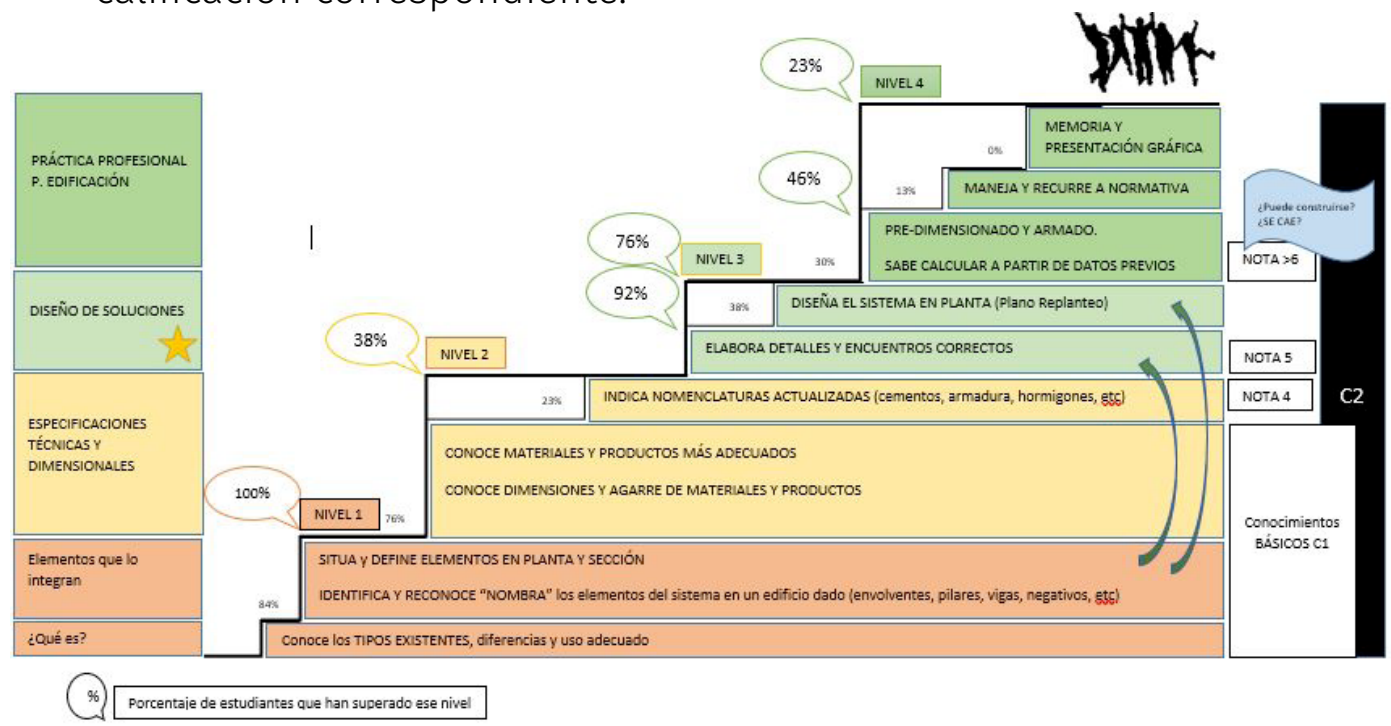

Figura 5. Escalera de aprendizaje aplicada al tema Forjados.

Los resultados obtenidos reflejan los conocimientos que presenta el estudiante al inicio "To" y al final "Tf" de las sesiones. La Tabla 1 compara gráficamente estos resultados respecto a los distintos apartados de contenidos establecidos en cada tema. A este esquema se han incorporado los obtenidos tras la revisión de la Práctica de Curso (entrega final "PC") al considerarla relevante por su obligatoriedad en la asignatura, representativa del trabajo no presencial realizado por el estudiante a partir de los conocimientos adquiridos en el aula y, como reflejo de los procedimientos y valores adquiridos con vistas a la práctica profesional.

Esta tabla comparativa permite observar, entre otros, cómo los resultados de las actividades resueltas en el aula coinciden con un mejor resultado en los cálculos que el estudiante realiza para la resolución de la Práctica de Curso. Con vistas a un nuevo diseño docente, se han 
podido extraer los apartados en los que el estudiante presenta mayor dificultad para resolver de forma autónoma (como las actividades resueltas en casa sin la guía o pautas del profesor).

Tabla 1. Comparativa entre resultados de aprendizaje To$\mathrm{Tf}-\mathrm{PC}$

\begin{tabular}{|c|c|c|c|c|c|c|c|c|c|c|c|c|c|}
\hline \multicolumn{2}{|c|}{ APARTADOS A EVALUAR } & \multicolumn{3}{|c|}{ CERRAMIENTOS } & \multicolumn{3}{|c|}{ CUBIERTAS } & \multicolumn{3}{|c|}{ FORJADOS } & \multicolumn{3}{|c|}{ CIMIENTOS } \\
\hline & & To & If & Pc & To & If & Pc & To & If & Pc & To & Tf & Pc \\
\hline \multirow{2}{*}{$\begin{array}{l}\text { PRÁCTICA } \\
\text { PROFESIONAL }\end{array}$} & MEMORIA & & & & & & & & & & & & \\
\hline & PLANOS & & & & & & & & & & & & \\
\hline \multirow[t]{3}{*}{$\begin{array}{l}\text { DISEÑO DE } \\
\text { SOLUCIONES }\end{array}$} & $\begin{array}{l}\text { PREDIMENSIONADO } \\
\text { ARMADO }\end{array}$ & & & & & & & & & & & & \\
\hline & $\begin{array}{l}\text { DETALLES } \\
\text { CONSTRUCTIVOS }\end{array}$ & & & & & & & & & & & & \\
\hline & PLANTAS & & & & & & & & & & & & \\
\hline \multirow[t]{3}{*}{ ESPECIFICACIONES } & NOMENCLATURAS & & & & & & & & & & & & \\
\hline & DIMENSIONES & & & & & & & & & & & & \\
\hline & $\begin{array}{l}\text { MATERIALES } \\
\text { PRODUCTOS }\end{array}$ & & & & & & & & & & & & \\
\hline $\begin{array}{l}\text { CONOCIMIENTOS } \\
\text { PREVIOS }\end{array}$ & $\begin{array}{l}\text { OBJETIVOS } \\
\text { TIPOS } \\
\text { ELEMENTOS }\end{array}$ & & & & & & & & & & & & \\
\hline
\end{tabular}

\section{Evaluación del CMD aplicado}

\section{Valoración de la experiencia}

A final de cada tema se han podido extraer algunas de las dificultades detectadas por los estudiantes en el alcance de los objetivos marcados por la asignatura, conceptos umbral y puntos destacados en el aprendizaje a 
partir de las cuestiones incluidas. Asimismo, a fin de extraer su opinión sobre la metodología docente utilizada, se ha incorporado un cuestionario final a la asignatura ( $\mathrm{Fi}$ gura 6) que incluye consultas de interés metodológico a fin de evaluar la docencia y su relación con los resultados del estudiante al final del cuatrimestre.

A las cuestiones planteadas, las respuestas obtenidas confirman el interés despertado en el estudiante: El 85\% de los estudiantes considera que la mayor parte de los contenidos conceptuales y procedimentales los ha aprendido durante la resolución de las actividades prácticas en el aula. Asimismo, el 60\% añade que las correcciones de las prácticas semanales en la pizarra han favorecido el aprendizaje. De igual forma, las muestras de prácticas y exámenes de años anteriores les resulta de mucha utilidad. Entre los comentarios extraídos de la evaluación de la docencia, se expresa el interés y utilidad de continuar con la metodología experimentada aumentando las actividades prácticas y resolución de problemas en clase. El $100 \%$ de los asistentes solicita, entre las posibles mejoras, aumentar el tiempo destinado a la resolución de estas prácticas en clase profundizando en la definición de especificaciones técnicas y dimensionales de los materiales y productos utilizados.

La "barra de estado" adjunta trata de reflejar el grado de satisfacción del estudiante con el nivel de aprendizaje adquirido de cara al examen final. Los datos obtenidos se muestran en la Figura 7 comparando los resultados antes y después de dicha prueba.

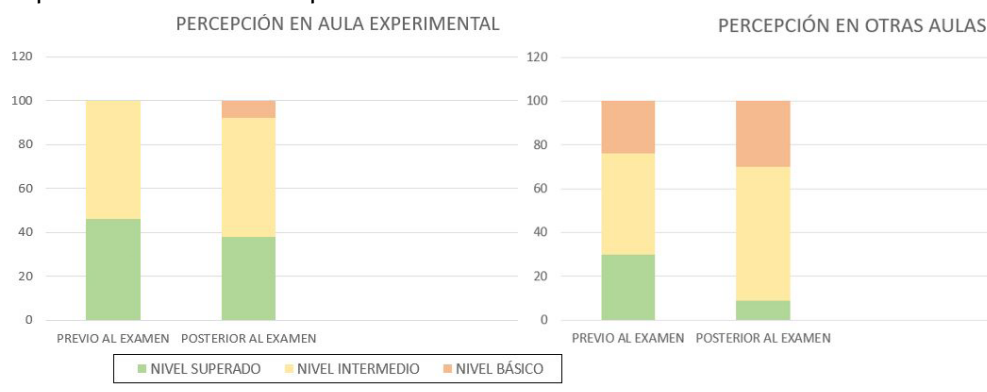

Figura 6. Cuestionario final de evaluación de la docencia

Jornadas de Formación e Innovación Docente del Profesorado I № 1 (2018) Esta obra se distribuye con la licencia Creative Commons 


\section{EVALUACIÓN DE LA DOCENCIA}

¿Crees que los temas impartidos en la asignatura son útiles para tu formación como Arquitecto?

$\mathrm{SI} / \mathrm{NO}$

¿Te ha costado llevar la asignatura hasta el final?

Durante el desarrollo de la asignatura, califica de 1 a 5 en qué momentos consideras que has aprendido mejor:

ASPECTO A VALORAR
\begin{tabular}{|l|c|c|c|c|c|}
\hline & $\begin{array}{c}\text { SIEMPRE } \\
\mathbf{5}\end{array}$ & $\begin{array}{c}\text { A MENUDO } \\
\mathbf{4}\end{array}$ & $\begin{array}{c}\text { A VECES } \\
\mathbf{3}\end{array}$ & $\begin{array}{c}\text { POCAS VECES } \\
\mathbf{2}\end{array}$ & $\begin{array}{c}\text { NUNCA } \\
\mathbf{1}\end{array}$ \\
\hline DURANTE LAS CLASES TEÓRICAS TRADICIONALES & & & & & \\
\hline DURANTE LA RESOLUCIÓN DE ACTIVIDADES EN CLASE & & & & & \\
\hline REALIZANDO LAS PRÁCTICAS EN CASA & & & & & \\
\hline CORRIGIENDO MI PRÁCTICA DE LA PIZARRA & & & & & \\
\hline UTILIZANDO LOS P. POINT DE LA ASIGNATURA eV3 & & & & & \\
\hline UTILIZANDO LA WEB DEL PROFESOR & & & & & \\
\hline
\end{tabular}

Menciona algunos conceptos cuyo aprendizaje te haya producido especiales dificultades.

¿Qué causas explican esas dificultades detectadas?

¿Cómo mejorarías ese aprendizaje?

En general, ¿¿Qué mejorarías de la docencia que has recibido?

\section{NIVEL DE APRENDIZAJE ADQUIRIDO}

Con vistas a la evaluación final, señala en la barra adjunta el NIVEL DE PREPARACIÓN con el que consideras que llegas al examen

\begin{tabular}{|l|l|l|}
\hline NIVEL INICIAL & NIVEL INTERMEDIO & NIVEL SUPERADO \\
\hline
\end{tabular}

Figura 7: Percepción del estudiante sobre su nivel de preparación. 2018

\section{Conclusiones finales}

Tras la obtención de calificaciones de la primera convocatoria, se pueden reflejar gráficamente los resultados del aprendizaje del CMD propuesto y aplicado sobre la asignatura de Construcción 2 durante el curso 2018.

Tal y como puede apreciarse en la Figura 8, los resultados obtenidos en la evaluación del curso muestran un importante avance en la asimilación de contenidos respecto 
a cursos anteriores y, sobre todo, una mayor fluidez en la argumentación crítica de los resultados. El alto porcentaje de éxito se refleja en aquellos estudiantes que, asistiendo a clase, han seguido la dinámica establecida y como resultado, el $84 \%$ de los alumnos implicados la han superado en esta primera convocatoria lo que refuerza la metodología aplicada para el aprendizaje en el aula a partir de actividades y resolución de cuestiones que lo acercan a su práctica profesional.

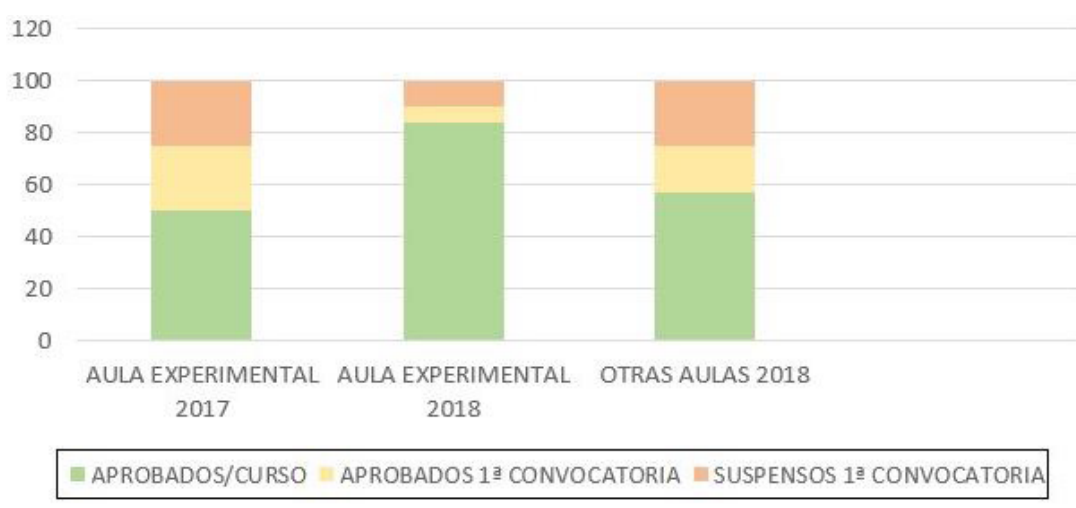

Figura 8. Comparativa de resultados de la evaluación continua.

Como curiosidad, los resultados obtenidos tras las calificaciones finales de la asignatura contrastan con la percepción previa del estudiante sobre su nivel de preparación por lo que convendría reflexionar sobre la inseguridad del estudiante y la realidad de su percepción ante una prueba de evaluación siendo conscientes de la complejidad y exigencias en la materia.

\section{Nuevas líneas de mejora y CMD}

Con vistas a un nuevo CMD en 2019, se pretende incorporar mejoras en la docencia y desarrollo de las actividades a partir de las incidencias o dificultades detectadas tal y como se expone en el apartado 3.1. (Valoración sobre el desarrollo de las sesiones): 
Se prevé completar la mejora de la asignatura con la entrega de los enunciados de actividades, a los grupos de trabajo, para centrar la disposición del profesor como tutor y favorecer la autonomía sin continuas interrupciones durante el desarrollo de los ejercicios. En esta línea, las tablas e información normativa deberá aportarlas el estudiante.

Se reducirán y concretarán nuevos P. Point para evitar el desorden que lleva ir pasando diapositivas de un lado a otro. Se ordenarán en función de su necesidad/utilidad en el desarrollo de las explicaciones.

Las actividades del aula se realizarán a mano, no a ordenador. Apoyando la expresión gráfica del estudiante.

Se controlará la asistencia con las entregas y actividades en clase, no se pasará lista.

Respecto al desarrollo de la Práctica de Curso, se ha reflejado la dificultad del estudiante para elaborar la memoria descriptiva y justificativa como parte del proyecto de ejecución completo por lo que, en clase, se incluirán actividades que revisen este aspecto. Asimismo, se destinará tiempo en las correcciones de la práctica a la definición de especificaciones técnicas y dimensionales de los materiales y productos utilizados.

En cualquier caso, se han presentado dificultades para representar los resultados de aprendizaje en una escalera de progresión del aprendizaje a partir de las actividades y test desarrollados por lo que, para el próximo curso y CMD, se propone llevar a cabo un desarrollo más específico de las escaleras de aprendizaje, extensible a todo el temario, que permita llegar a una nueva propuesta de evaluación y calificación de los estudiantes. De estos resultados, se propondrá a la asignatura, un nuevo formato de examen. 


\section{Referencias bibliográficas}

Bain, K. (2004). Traducción Barberá, O. (2008). Lo que hacen los mejores profesores universitarios. Universidad de Valencia. España: PUV.

Bahón, J. (2016). Laboratorio Internacional de Innovación y Coaching Educativo. Recuperado de https://www.tuinnovas.com.

Finkel, D. Traducción Barberá, O. (2008). Dar clases con la boca cerrada. Valencia. España: Universidad de valencia.

Libro de resúmenes del 1 Congreso Internacional de Innovación y Tendencias Educativas. INNTED (2017). Innovación y Tendencias Educativas. Sevilla. España: Universidad de Sevilla.

Porlán, R. (2017). Enseñanza Universitaria. Como mejorarla. Universidad de Sevilla. España: Ediciones Morata.

Proyecto de Innovación Docente II Plan propio de Docencia. Departamento de Estructuras de la Edificación e Ingeniería del Terreno (Convocatoria 2016). El aprendizaje de conceptos umbral mediante tics en un entorno multidisciplinar. Universidad de Sevilla.

Proyecto de Innovación Docente III Plan propio de Docencia. Departamento de Construcción Arquitectónica 1 (Convocatoria 2017). Tutorización de las horas de aprendizaje no presencial mediante tics en disciplinas técnicas. Universidad de Sevilla.

Jornadas de Formación e Innovación Docente del Profesorado | № 1 (2018) Esta obra se distribuye con la licencia Creative Commons 